\title{
Article
}

\section{Development of a MicroRNA Signature Predictive of Recurrence and Survival in Pancreatic Ductal Adenocarcinoma}

\author{
Nikhil T. Sebastian ${ }^{1}$, Amy Webb ${ }^{2}$, Kenneth W. Merrell ${ }^{3}$, Eugene J. Koay ${ }^{4}$, Adam R. Wolfe ${ }^{5}$, Lizhi Zhang ${ }^{6}$, \\ Tyler J. Wilhite ${ }^{7}$, Dalia Elganainy ${ }^{4}{ }^{(0)}$, Ryan Robb ${ }^{8}$, Wei Chen ${ }^{9}$, Jordan Cloyd ${ }^{10}$, Mary Dillhoff ${ }^{10}$, Allan Tsung ${ }^{10}$, \\ Laith Abushahin ${ }^{11} \mathbb{D}$, Anne Noonan ${ }^{11}$ and Terence M. Williams ${ }^{12, *(\mathbb{D})}$
}

\section{check for} updates

Citation: Sebastian, N.T.; Webb, A.; Merrell, K.W.; Koay, E.J.; Wolfe, A.R.; Zhang, L.; Wilhite, T.J.; Elganainy, D.; Robb, R.; Chen, W.; et al.

Development of a MicroRNA Signature Predictive of Recurrence and Survival in Pancreatic Ductal Adenocarcinoma. Cancers 2021, 13, 5168. https://doi.org/10.3390/ cancers 13205168

Academic Editor: Julien Taieb

Received: 19 August 2021

Accepted: 6 October 2021

Published: 15 October 2021

Publisher's Note: MDPI stays neutral with regard to jurisdictional claims in published maps and institutional affiliations.

Copyright: (c) 2021 by the authors. Licensee MDPI, Basel, Switzerland. This article is an open access article distributed under the terms and conditions of the Creative Commons Attribution (CC BY) license (https:/ / creativecommons.org/licenses/by/ $4.0 /)$.
1 Department of Radiation Oncology, Emory University Winship Cancer Institute, 1365 Clifton Rd, Atlanta, GA 30322, USA; nikhil.sebastian@emory.edu

2 Department of Biomedical Informatics, The Ohio State University College of Medicine, 320 Lincoln Tower, 1800 Cannon Drive, Columbus, OH 43210, USA; Amy.Hite@osumc.edu

3 Department of Radiation Oncology, Mayo Clinic, 200 First St SW, Rochester, MN 55090, USA; Merrell.Kenneth@mayo.edu

4 Department of Radiation Oncology, The University of Texas MD Anderson Cancer Center, 1400 Pressler St, Houston, TX 77030, USA; EKoay@mdanderson.org (E.J.K.); dalia_elganainy@dfci.harvard.edu (D.E.)

5 Department of Radiation Oncology, The Winthrop P. Rockefeller Cancer Institute, The University of Arkansas for Medical Sciences, Little Rock, AR 72205, USA; awolfe@uams.edu

6 Division of Anatomic Pathology, Mayo Clinic, 200 First St SW, Rochester, MN 55905, USA; zhang.lizhi@mayo.edu

7 Department of Radiation Oncology, UPMC Hillman Cancer Center-Shadyside, 55230 Centre Ave, Pittsburgh, PA 15232, USA; Wilhitetj2@upmc.edu

8 Department of Pathology, University of North Carolina, Chapel Hill, NC 27599, USA; robb28@email.unc.edu

9 Department of Pathology, The Ohio State University, 450 W. 10th Ave, Columbus, OH 43210, USA; wei.chen2@osumc.edu

10 Division of Surgical Oncology, Department of Surgery, The Ohio State University Comprehensive Cancer Center-Arthur G. James Cancer Hospital and Richard J. Solove Research Institute, 460 W. 10th Ave, Columbus, OH 43210, USA; jordan.cloyd@osumc.edu (J.C.); mary.dillhoff@osumc.edu (M.D.); allan.tsung@osumc.edu (A.T.)

11 Division of Medical Oncology, Department of Internal Medicine, The Ohio State University Comprehensive Cancer Center-Arthur G. James Cancer Hospital and Richard J. Solove Research Institute, 460 W. 10th Ave, Columbus, OH 43210, USA; laith.abushahin@osumc.edu (L.A.); anne.noonan@osumc.edu (A.N.)

12 Department of Radiation Oncology, City of Hope National Medical Center, 1500 E. Duarte Rd, Duarte, CA 91010, USA

* Correspondence: terwilliams@coh.org; Tel.: +1-(262)-218-2332; Fax: +1-(626)-218-5334

Simple Summary: Optimal patient selection for radiotherapy in pancreatic cancer is unestablished and may be improved with molecular profiling. To this end, we developed and validated a microRNA signature that predicted for worse locoregional recurrence and overall survival in patients with resectable pancreatic cancer. In a separate cohort of patients with borderline resectable and locally advanced pancreatic cancer, this risk signature was also predictive of worse locoregional recurrence, distant recurrence, and overall survival. Additionally, borderline resectable or locally advanced patients who had high risk score and did not receive radiation had worse outcomes compared to patients who either had low risk score or received radiation, irrespective of risk score. This risk signature may be useful in assessing patient prognosis and tailor therapy in patients with resectable, borderline resectable, or locally advanced pancreatic cancer, but requires further study.

Abstract: Background: Optimal patient selection for radiotherapy in pancreatic ductal adenocarcinoma (PDAC) is unestablished. Molecular profiling may select patients at high risk for locoregional recurrence (LRR) who would benefit from radiation. Methods: We included resectable pancreatic cancer (R-PDAC) patients, divided into training and validation cohorts, treated among three institutions with surgery and adjuvant chemotherapy, and borderline resectable or locally advanced pancreatic cancer (BR/LA-PDAC) patients treated with chemotherapy with or without radiation at the primary study institution. We isolated RNA from R-PDAC surgical specimens. Using NanoString, we identified miRNAs differentially expressed between normal and malignant pancreatic 
tissue. ElasticNet regression identified two miRNAs most predictive of LRR in the training cohort, $\mathrm{miR}-181 \mathrm{~b} / \mathrm{d}$ and $\mathrm{miR}-575$, which were used to generate a risk score (RS). We evaluated the association of the median-dichotomized RS with recurrence and overall survival (OS). Results: We identified 183 R-PDAC and 77 BR/LA-PDAC patients with median follow up of 37 months treated between 2001 and 2014. On multivariable analysis of the R-PDAC training cohort $(n=90)$, RS was associated with worse LRR (HR $=1.34 ; 95 \% \mathrm{CI} 1.27-11.38 ; p=0.017)$ and $\mathrm{OS}(\mathrm{HR}=2.89 ; 95 \% \mathrm{CI}$ $1.10-4.76 ; p=0.027)$. In the R-PDAC validation cohort, RS was associated with worse LRR (HR = 2.39; 95\%CI 1.03-5.54; $p=0.042)$, but not OS ( $p=0.087)$. For BR/LA-PDAC, RS was associated with worse LRR (HR $=2.71 ; 95 \% C I 1.14-6.48 ; p=0.025)$, DR (HR = 1.93; 95\%CI 1.10-3.38; $p=0.022)$, and OS $(\mathrm{HR}=1.97 ; 95 \% \mathrm{CI} 1.17-3.34 ; p=0.011)$. Additionally, after stratifying by $\mathrm{RS}$ and receipt of radiation in BR/LA-PDAC patients, high RS patients who did not receive radiation had worse LRR $(p=0.018)$, DR $(p=0.006)$, and OS $(p<0.001)$ compared to patients with either low RS or patients who received radiation, irrespective of RS. Conclusions: RS predicted worse LRR and OS in R-PDAC and worse LRR, DR, and OS in BR/LA-PDAC. This may select patients who would benefit from radiation and should be validated prospectively.

Keywords: pancreatic cancer; microRNA; locoregional recurrence; local recurrence; adjuvant radiation; neoadjuvant radiation

\section{Introduction}

Pancreatic ductal adenocarcinoma (PDAC) is the third leading cause of cancer mortality in the United States, accounting for over 45,000 deaths annually [1]. PDAC is typified by high rates of local (e.g., primary tumor or tumor bed), regional (e.g., lymph nodes), and distant recurrence after surgery. While surgical resection offers the best chance for long-term outcomes, many patients do not initially present with resectable disease, but rather with borderline-resectable, locally advanced (unresectable), or metastatic disease. For resectable disease, the role of postoperative radiotherapy is controversial, given the uncertain benefit in overall survival in randomized trials [2-4]. The role of radiotherapy is similarly controversial in patients with borderline or locally advanced PDAC [5,6]. Despite the equivocal benefit of radiotherapy on survival in cohorts of unselected patients, it is clear that radiotherapy improves locoregional control [2-5]. However, predicting which patients might be at increased risk of locoregional failure is critical to determining whether certain subsets of patients benefit from additional locoregional therapy, given the potential toxicity from radiation. Molecular personalized assessment may allow for improved selection of patients who would benefit from radiotherapy [7].

Molecular assays predictive of recurrence are utilized in treatment selection for other malignancies with great clinical success [8,9]. Identification of novel biomarkers may aid in the prognostication or tailoring of treatment or follow up. Specifically, microRNAs (miRNAs) have emerged as valuable biomarkers; these non-coding RNAs are instrumental in post-transcriptional regulation of gene expression and interact with target mRNAs to induce their degradation. Their dysregulation has been identified in numerous cancers, and they are of particular interest in PDAC given their association with tumor invasiveness and treatment resistance [10]. Multiple miRNA expression profiling studies have established differential expression of miRNAs between normal and malignant pancreatic tissue [11]. These miRNAs have been implicated in pancreatic cancer progression through a variety of mechanisms, including negative regulation of tumor suppressor genes (e.g., miR-21mediated downregulation of PTEN), underexpression of tumor suppressing miRNAs (e.g., miR-124 negative regulation of Rac1 oncogene), or cell cycle alteration resulting in enhanced proliferation (e.g., miR-203 downregulation inducing G1 phase progression) [12]. In a previous single institution pilot study, we assessed the prognostic capacity of miRNAs and developed a tentative signature derived from a small cohort of patients [13]. In this current study, we sought to identify and then validate a revised miRNA signature predictive 
of locoregional recurrence in a cohort of patients with resected PDAC derived from a large, multi-institutional cohort. In a secondary analysis, we sought to confirm the predictive and prognostic utility of the miRNA signature in a cohort of patients with borderline resectable or locally advanced PDAC treated at our institution.

\section{Methods}

\subsection{Patient Selection and Endpoints}

This study was approved by the institutional review boards of all participating institutions (IRB protocol 2014C0077 (OSU-14093), opened in 2014). For patients with resected PDAC, we obtained core samples of formalin-fixed, paraffin-embedded (FFPE) tumor and adjacent normal tissue from patients who underwent surgery and adjuvant chemotherapy at Ohio State University, Mayo Clinic, or MD Anderson. For patients with borderline resectable or locally advanced PDAC, FFPE tumors from pretreatment core biopsy samples were obtained. All patients with borderline resectable or locally advanced PDAC were treated with definitive intent at Ohio State University with chemotherapy, with or without radiotherapy or surgery.

Recurrence was defined radiologically using computed tomography (CT) scans of the abdomen and pelvis during follow up, which was performed every 3-6 months after completion of treatment. Locoregional (local and regional) recurrence was defined as either pathologically confirmed recurrence within the surgical bed (for resected PDAC), primary tumor (for borderline-resectable or locally advanced PDAC), or lymph nodes occurring in standard post-operative radiation treatment fields, or as measurable progression of disease in these regions on 2 consecutive scans per RECIST version 1.1 criteria [14] with corroborating rise in CA19-9. Distant recurrence was defined as recurrence outside of the surgical bed/pancreas, and regional lymph nodes. Overall survival was defined as the interval between the date of surgery (for resected PDAC) or first induction chemotherapy cycle (for borderline resectable and locally advanced PDAC) and the date of death or last follow up.

\subsection{MiRNA Expression Profiling}

For all samples, we used the Norgen FFPE RNA Isolation kit (Norgen Biotek, Thorold, ON, Canada) to collect total genomic RNA from viable, non-necrotic regions of tumor in FFPE blocks as defined by board-certified pathologists specializing in gastrointestinal pathology as previously described [15]. Expression profiling of miRNAs was performed using the nCounter Human v3 miRNA Expression Assay (NanoString Technologies, Seattle WA, USA). Counts were normalized with voom and limma $\mathrm{R}$ packages to perform differential expression analysis between groups of samples.

A miRNA was removed if more than $90 \%$ of the samples had log counts less than the negative background. Negative background was calculated as the mean of the $\log 2$ negative background counts plus 1.5 times the standard deviation. A sample was removed if more than $70 \%$ of miRNA probes fell below the background cutoff for the Ohio State University cohort and $60 \%$ for the Mayo Clinic and MD Anderson cohorts. Final sample counts were 69, 26, and 88 for Mayo Clinic, MD Anderson, and Ohio State University, respectively. The filtered data were normalized by the geometric mean and $\log 2$ transformed.

With mixed modeling we identified miRNAs differentially expressed in tumor and normal tissue of resected PDAC patients. Using the R CrossValidate package, we created equal-sized training and validation cohorts of patients with resected PDAC balanced by institution and locoregional recurrence events. In the training cohort there were 43,34 , and 13 patients, and in the validation cohort there were 45, 35, and 13 patients treated at Ohio State University, Mayo Clinic, and MD Anderson Cancer Center, respectively. Using the pool of miRNAs distinguishing tumor from normal, ElasticNet regression was used to select predictive groups of miRNAs and identified two miRNAs (miR-181b/d and miR-575) predictive of locoregional recurrence (handled as a censored variable) and overall survival in the training dataset. Beta coefficients, generated by ElasticNet, were used to calculate a 
risk score by summing the product of each miRNA expression level and its beta coefficient, as follows:

$-1.8142 *$ hsa.miR.181b.5p/hsa.miR.181d.5p - 0.1856*hsa.miR.575

We studied the miRNA risk score both as a continuous variable and as a variable dichotomized by the respective median of each cohort and evaluated its association with locoregional recurrence, distant recurrence, and overall survival using Cox proportional hazards and Kaplan-Meier analysis. For resectable PDAC patients, we accounted for age, pathologic $\mathrm{T}$ and $\mathrm{N}$ stage, histologic grade, postoperative CA 19-9, and surgical margin status in the Cox model. For borderline resectable or locally advanced patients, we accounted for age, clinical $\mathrm{T}$ and $\mathrm{N}$ stage, receipt of radiation, and surgical resection status. All statistical analyses were performed using R version 3.5.0 and 3.4.3 (R Project for Statistical Computing).

\section{Results}

\subsection{Resectable Pancreatic Cancer}

We identified a total of 183 resectable PDAC patients who underwent surgical resection followed by chemotherapy. The median follow up of all patients was 20.9 months (interquartile range (IQR) 13.6-33.3), and the median follow up of living patients was 37.1 months (IQR 25.3-55.3). Of these, 90 patients were assigned to the training cohort and 93 were assigned to the validation cohort (Table 1).

Table 1. Patient and disease characteristics of patients with resectable pancreatic cancer in the training and validation cohorts.

\begin{tabular}{|c|c|c|c|c|c|c|}
\hline \multirow{2}{*}{ Variable } & \multicolumn{3}{|c|}{ Training Cohort } & \multicolumn{3}{|c|}{ Validation Cohort } \\
\hline & $\begin{array}{c}\text { Low Risk } \\
(n=45)\end{array}$ & $\begin{array}{l}\text { High Risk } \\
(n=45)\end{array}$ & $p$ & $\begin{array}{l}\text { Low Risk } \\
(n=46)\end{array}$ & $\begin{array}{l}\text { High Risk } \\
(n=47)\end{array}$ & $p$ \\
\hline \multicolumn{7}{|l|}{ Age (years) } \\
\hline$<60$ & $15(33.3 \%)$ & $12(26.7 \%)$ & \multirow{2}{*}{0.65} & $15(32.6 \%)$ & $13(27.7 \%)$ & \multirow{2}{*}{0.60} \\
\hline$\geq 60$ & $30(66.7 \%)$ & $33(73.3 \%)$ & & $31(67.4 \%)$ & $34(72.3 \%)$ & \\
\hline \multicolumn{7}{|l|}{ Sex } \\
\hline Male & $24(53.3 \%)$ & $27(60.0 \%)$ & \multirow{2}{*}{0.67} & $25(54.3 \%)$ & $28(59.6 \%)$ & \multirow{2}{*}{0.61} \\
\hline Female & $21(46.7 \%)$ & $18(40.0 \%)$ & & $21(45.7 \%)$ & $19(40.4 \%)$ & \\
\hline \multicolumn{7}{|l|}{$\begin{array}{l}\text { Pathologic } \mathrm{T} \\
\text { stage }\end{array}$} \\
\hline 1-2 & $12(26.7 \%)$ & $4(8.9 \%)$ & \multirow{2}{*}{0.05} & $8(17.4 \%)$ & $4(8.5 \%)$ & \multirow{2}{*}{0.20} \\
\hline $3-4$ & $33(73.3 \%)$ & $41(91.1 \%)$ & & $38(82.6 \%)$ & $43(91.5 \%)$ & \\
\hline \multirow{2}{*}{\multicolumn{7}{|c|}{$\begin{array}{l}\text { Pathologic } \mathrm{N} \\
\text { stage }\end{array}$}} \\
\hline & & & & & & \\
\hline 0 & $14(31.1 \%)$ & $9(20.0 \%)$ & \multirow{2}{*}{0.33} & $17(37.0 \%)$ & $8(17.0 \%)$ & \multirow{2}{*}{0.03} \\
\hline 1 & $31(68.9 \%)$ & $36(80.0 \%)$ & & $29(63.0 \%)$ & $39(83.0 \%)$ & \\
\hline \multicolumn{7}{|l|}{ Margins } \\
\hline Negative & $35(77.8 \%)$ & $17(37.8 \%)$ & \multirow{2}{*}{$<0.001$} & $37(80.4 \%)$ & $16(34.0 \%)$ & \multirow{2}{*}{$<0.001$} \\
\hline Positive & $10(22.2 \%)$ & $28(62.2 \%)$ & & $9(19.6 \%)$ & $31(66.0 \%)$ & \\
\hline \multicolumn{7}{|l|}{ Grade } \\
\hline $1-2$ & $19(42.2 \%)$ & $28(62.2 \%)$ & \multirow{2}{*}{0.09} & $18(39.1 \%)$ & $27(57.4 \%)$ & \multirow{2}{*}{0.08} \\
\hline 3 & $26(57.8 \%)$ & $17(37.8 \%)$ & & $28(60.9 \%)$ & $20(42.6 \%)$ & \\
\hline \multirow{2}{*}{\multicolumn{7}{|c|}{$\begin{array}{c}\text { Post-op CA } \\
19-9\end{array}$}} \\
\hline & & & & & & \\
\hline$\leq 90$ & $30(66.7 \%)$ & $24(21.0 \%)$ & \multirow{3}{*}{1.00} & $27(58.7 \%)$ & $27(57.4 \%)$ & \multirow{3}{*}{0.12} \\
\hline$>90$ & $7(15.6 \%)$ & $16(35.6 \%)$ & & $3(6.5 \%)$ & $9(19.1 \%)$ & \\
\hline Unavailable & $8(17.8 \%)$ & $16(35.6 \%)$ & & $16(34.8 \%)$ & $11(23.4 \%)$ & \\
\hline \multicolumn{7}{|l|}{$\begin{array}{l}\text { Locoregional } \\
\text { recurrence }\end{array}$} \\
\hline No & $36(80.0 \%)$ & $18(40.0 \%)$ & \multirow{2}{*}{$<0.001$} & $32(69.6 \%)$ & $23(48.9 \%)$ & \multirow{2}{*}{0.04} \\
\hline Yes & $9(20.0 \%)$ & $27(60.0 \%)$ & & $14(30.4 \%)$ & $24(51.1 \%)$ & \\
\hline
\end{tabular}


In the training cohort $(n=90)$, when analyzed as a continuous variable, the miR risk score was associated with locoregional recurrence (HR $=1.43 ; 95 \%$ CI 1.14-1.81; $p=0.002)$, but not distant recurrence (HR $=1.11 ; 95 \%$ CI $0.92-1.34 ; p=0.28)$ or overall survival $(\mathrm{HR}=1.07 ; 95 \%$ CI $0.91-1.25 ; p=0.44)$. After dichotomization, high miR risk score was associated with increased locoregional recurrence $(\mathrm{HR}=3.29 ; 95 \% \mathrm{CI} 1.53-7.04 ; p=0.002)$, but not distant recurrence (HR $=1.52 ; 95 \%$ CI $0.90-2.54 ; p=0.11)$. Overall survival was numerically worse but statistically non-significant in the high-risk group ( $\mathrm{HR}=1.58$; $95 \%$ CI $0.98-2.54 ; p=0.059$ ). Three-year locoregional control was $68.8 \%$ and $21.5 \%$ (logrank $p=0.0012$ ) for high- and low-risk groups, respectively, and 3-year overall survival was $45.5 \%$ and $20.3 \%$ (logrank $p=0.057$ ), respectively (Figure $1 \mathrm{~A}-\mathrm{C}$ ). On multivariable analysis, high risk score continued to be independently associated with locoregional recurrence $(\mathrm{HR}=1.34 ; 95 \%$ CI 1.27-11.38; $p=0.017)$ (Table S1). A higher miR risk score was also significantly associated with worse overall survival ( $\mathrm{HR}=2.89 ; 95 \%$ CI $1.10-4.76 ; p=0.027$ ), despite no significant association with distant recurrence (HR $=1.62 ; 95 \%$ CI $0.75-1.50$; $p=0.22$ ). In an analysis including receipt of radiation as a covariable (five patients received radiation), binary risk score remained associated with locoregional recurrence $(\mathrm{HR}=5.66$; 95\% CI 1.62-19.77; $p=0.007$ ) and overall survival ( $\mathrm{HR}=2.40 ; 95 \%$ CI 1.12-5.16; $p=0.025)$, and was not associated with distant recurrence ( $\mathrm{HR}=1.86$; 95\% CI 0.82-4.22; $p=0.14$ ). Finally, in a secondary multivariable analysis excluding the five patients who received adjuvant radiation in the training cohort, binary risk score remained associated with locoregional recurrence $(\mathrm{HR}=4.57 ; 95 \% \mathrm{CI} 1.35-15.49 ; p=0.015)$ and overall survival $(\mathrm{HR}=2.29 ; 1.08-4.89 ; p=0.031)$.

(A)

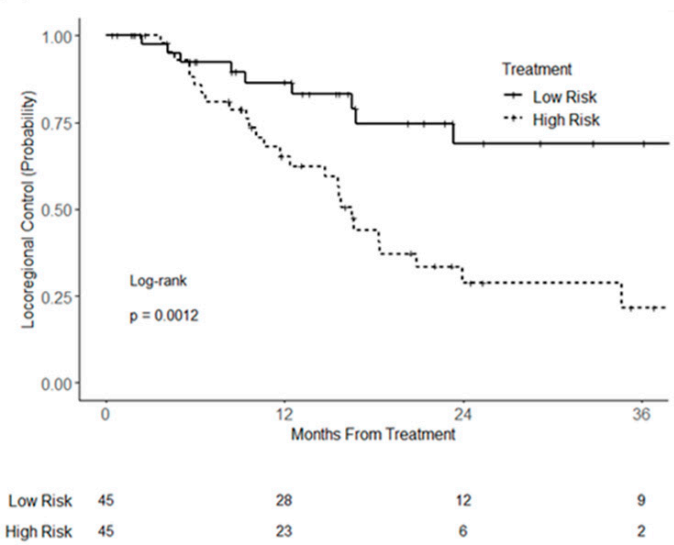

(B)

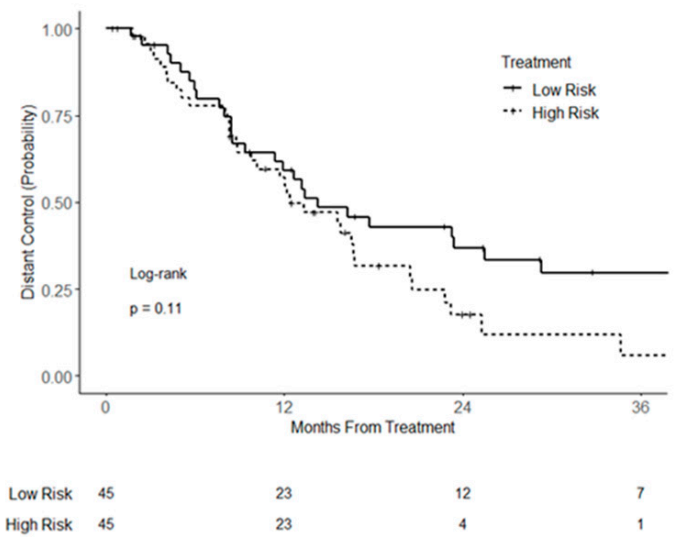

(C)

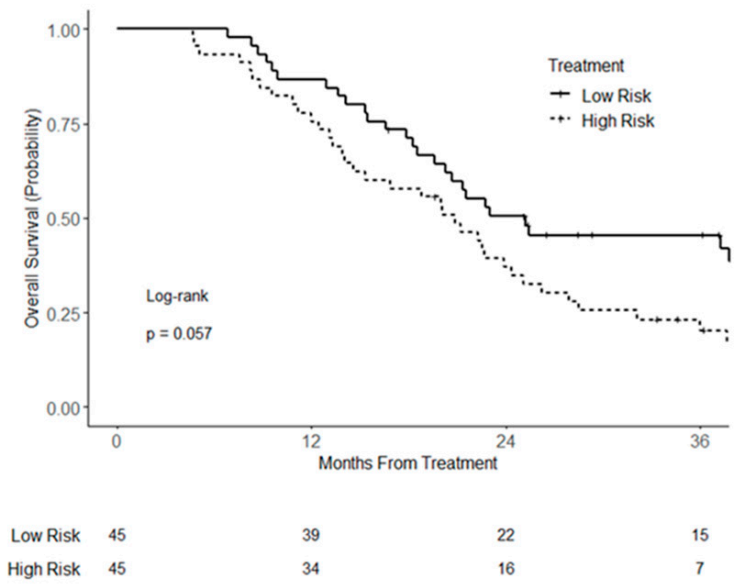

Figure 1. Kaplan-Meier curves of (A) locoregional recurrence, (B) distant recurrence, and (C) overall survival of the training cohort of resectable pancreatic cancer patients, stratified by risk score group. 
In the validation cohort $(n=93)$, high miR risk score (analyzed as a continuous variable) was associated with worse locoregional recurrence (HR $=1.27 ; 95 \%$ CI 1.00-1.60; $p=0.046)$ and overall mortality (HR $=1.21 ; 95 \%$ CI 1.01-1.44; $p=0.037)$ on univariable analysis. There was no significant association with distant recurrence $(\mathrm{HR}=1.05 ; 95 \% \mathrm{CI}$ $0.86-1.29 ; p=0.65)$. After dichotomization, high miR risk score was associated with worse locoregional recurrence $(\mathrm{HR}=2.08 ; 95 \%$ CI 1.07-4.03; $p=0.030)$ and overall survival $(\mathrm{HR}=1.61 ; 95 \% \mathrm{CI} 1.02-2.54 ; p=0.042)$, but not distant recurrence $(\mathrm{HR}=1.48 ; 95 \% \mathrm{CI}$ $0.87-2.52 ; p=0.15)$. Three-year locoregional control was $61.5 \%$ and $37.2 \%$ (logrank $p=0.027)$ for high- and low-risk groups, respectively, and 3-year overall survival was $33.5 \%$ and $15.0 \%$ (logrank $p=0.040$ ), respectively (Figure $2 \mathrm{~A}-\mathrm{C}$ ). On multivariable analysis, high miR risk score remained associated with increased locoregional recurrence $(\mathrm{HR}=2.39 ; 95 \% \mathrm{CI}$ 1.03-5.54; $p=0.042$ ) (Table S2), but not distant recurrence (HR = 1.83; 95\% CI 0.78-4.30; $p=0.16$ ). High risk score was numerically associated with worse overall survival, although this was statistically non-significant $(\mathrm{HR}=1.76 ; 95 \% \mathrm{CI} 0.92-3.36 ; p=0.087)$. In a secondary multivariable analysis excluding the two patients who received adjuvant radiation in the validation cohort, binary risk score trended toward significance for association with locoregional recurrence ( $\mathrm{HR}=2.04 ; 95 \%$ CI $0.88-4.75 ; p=0.097)$ but was significantly associated with worse overall survival $(\mathrm{HR}=1.59 ; 95 \% \mathrm{CI} 0.84-3.04 ; p=0.016)$.

(A)

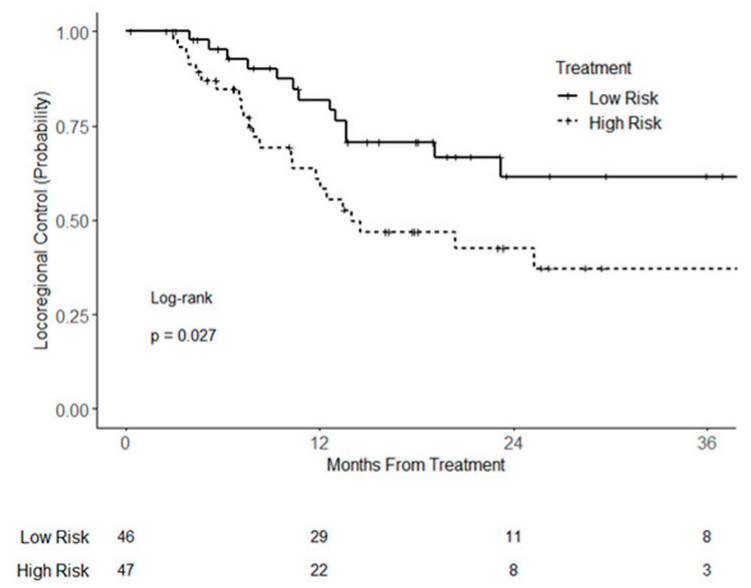

(C)

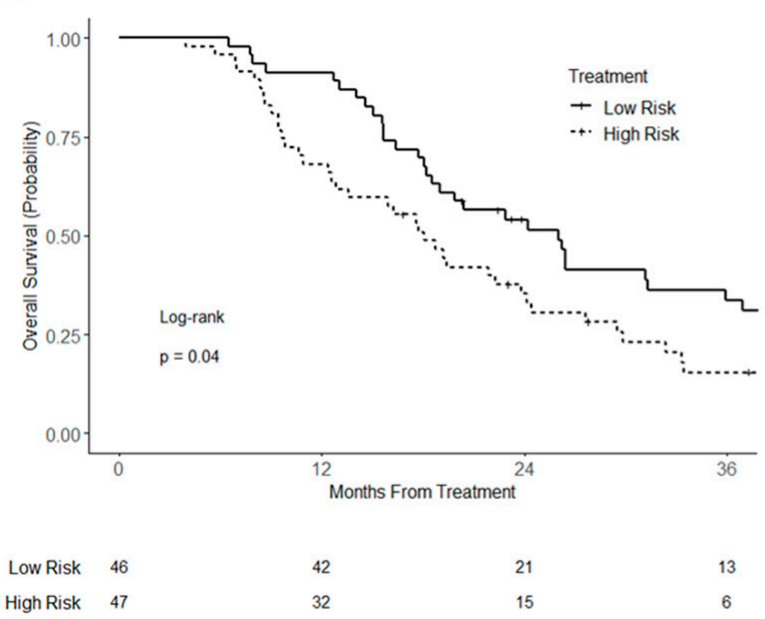

(B)

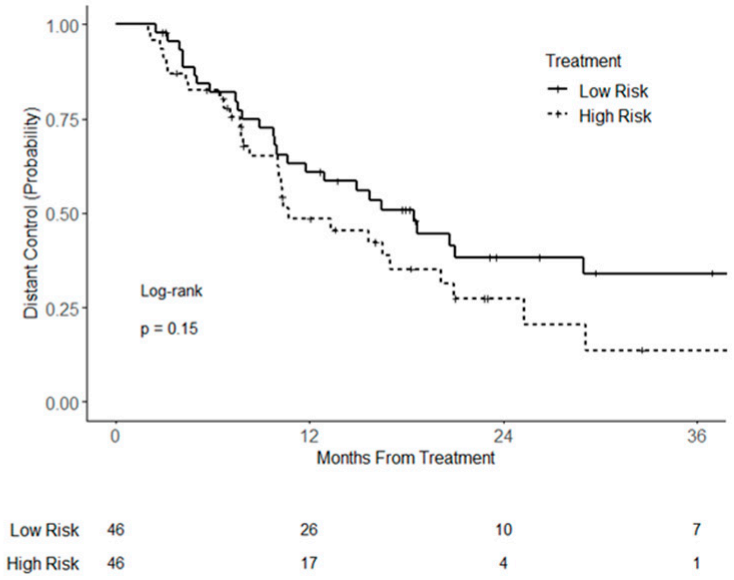

Figure 2. Kaplan-Meier curves of (A) locoregional control, (B) distant control, and (C) overall survival of the validation cohort of resectable pancreatic cancer patients, stratified by risk score group. 


\subsection{Borderline Resectable/Unresectable Pancreatic Cancer}

To evaluate the applicability of the miR risk signature to the neoadjuvant (borderline resectable) or inoperable setting (locally advanced), we identified a total of 77 patients with borderline resectable or locally advanced PDAC treated with chemotherapy with or without radiotherapy (Table S3). The median follow up of all patients was 13.8 months (IQR 11.1-31.5), and the median follow up of living patients was 37.3 months (33.5-42.7).

On univariable analysis, continuous miR risk score was associated with worse overall survival ( $\mathrm{HR}=1.32 ; 95 \% \mathrm{CI} 1.04-1.67 ; p=0.025)$ and distant recurrence $(\mathrm{HR}=1.32$; $95 \%$ CI $1.04-1.69 ; p=0.025)$. High miR risk score was associated with statistically nonsignificant increased locoregional recurrence ( $\mathrm{HR}=1.44 ; 95 \% \mathrm{CI} 0.97-2.14 ; p=0.072)$. After dichotomization, high binary miR risk score was associated with worse overall survival $(\mathrm{HR}=1.79 ; 95 \% \mathrm{CI} 1.11-2.90 ; p=0.018)$ and worse distant recurrence $(\mathrm{HR}=1.84 ; 95 \% \mathrm{CI}$ $1.07-3.15 ; p=0.027)$, and had a statistically non-significant association with higher locoregional recurrence $(\mathrm{HR}=2.09 ; 95 \% \mathrm{CI} 0.94-4.66 ; p=0.073$ ) (Figure 3A-C). On multivariable analysis, high risk score was associated with worse locoregional recurrence (HR $=2.71$; 95\% CI 1.14-6.48; $p=0.025)$ (Table S4), distant recurrence (HR $=1.93 ; 95 \%$ CI 1.10-3.38; $p=0.022)$, and overall survival ( $\mathrm{HR}=1.97 ; 95 \%$ CI $1.17-3.34 ; p=0.011)$.

(A)

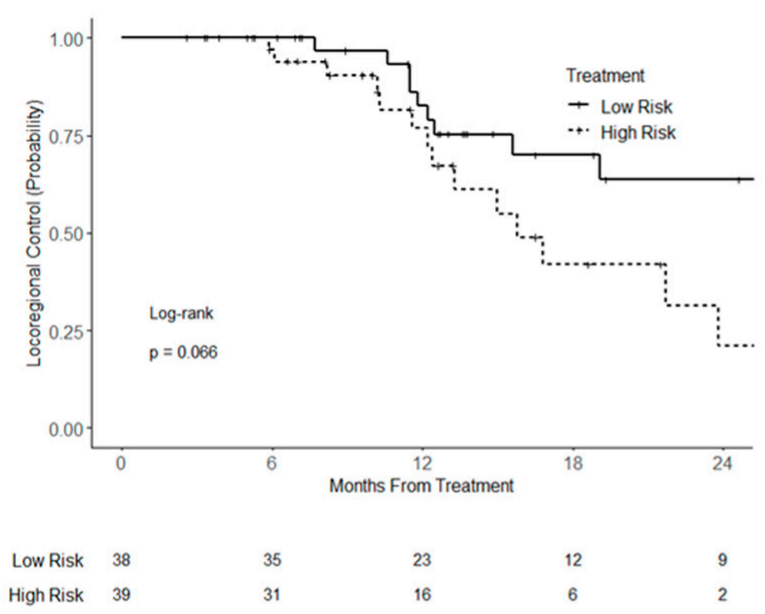

(B)

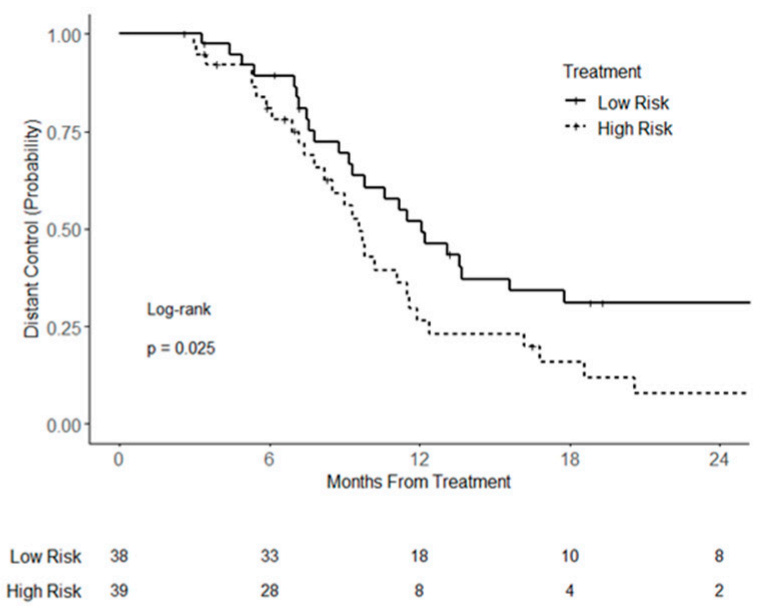

(C)

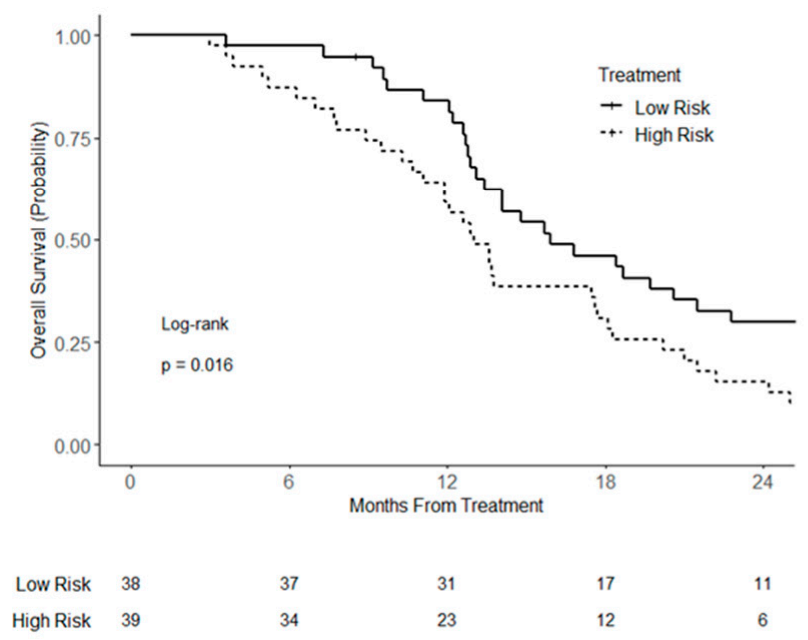

Figure 3. Kaplan-Meier curves of (A) locoregional control, (B) distant control, and (C) overall survival of patients with borderline resectable or locally advanced (unresectable) pancreatic cancer, stratified by risk score group. 
After stratification for risk score and receipt of radiotherapy, high-risk patients who did not receive radiotherapy had worse locoregional recurrence (log-rank $p=0.018)$, distant recurrence $(\log$-rank $p=0.006)$, and overall survival (log-rank $p=0.00029)$ (Figure 4 ).

(A)

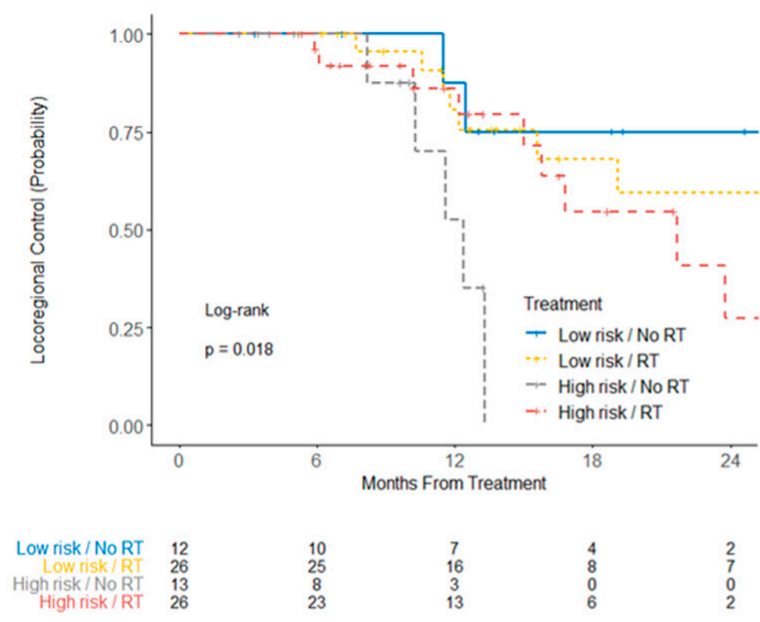

(B)

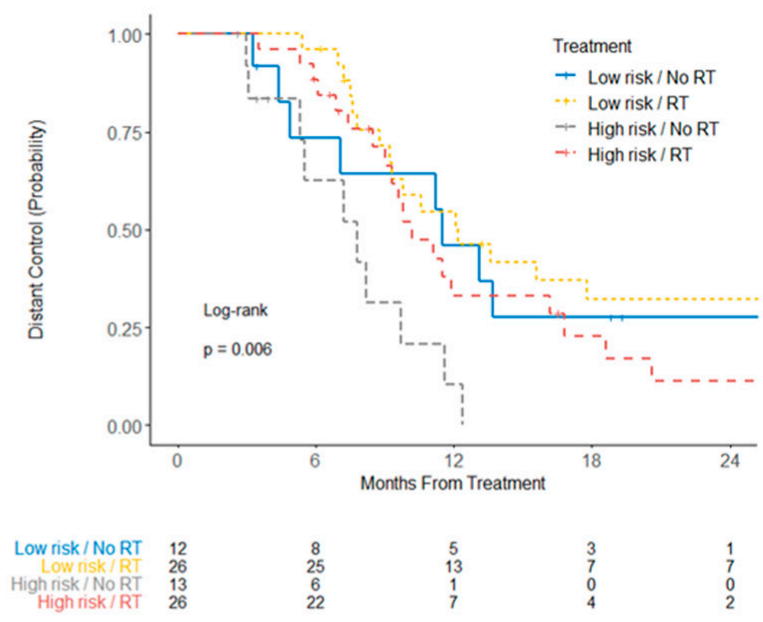

(C)

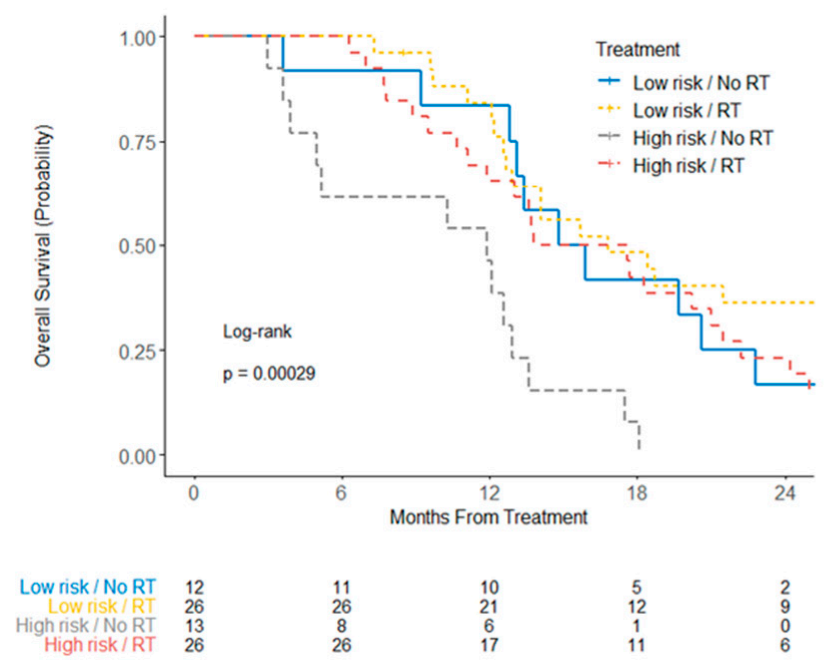

Figure 4. Kaplan-Meier curves of (A) locoregional control, (B) distant control, and (C) overall survival of patients with unresectable or borderline resectable pancreatic cancer, stratified by risk score group and receipt of radiation.

\section{Discussion}

We developed and validated a multi-miRNA risk score associated with higher risk of locoregional recurrence in a multi-institutional cohort of patients with resected PDAC. In addition, high miR risk score was frequently associated not only with locoregional recurrence, but also decreased survival. Furthermore, we found this miR risk score was associated with higher rates of locororegional recurrence, distant recurrence, and worse overall mortality in our single-institution cohort of patients with borderline resectable or locally advanced disease treated with chemotherapy with or without radiation. This is, to our knowledge, the first validated, molecular-based risk score developed for PDAC that can predict locoregional recurrence and survival.

During the past two decades, significant work has been undertaken to identify diverse molecular biomarkers for PDAC, but these studies have been hampered by small sample sizes and biomarker non-specificity [16]. MicroRNAs are small non-coding RNAs involved in post-transcriptional regulation of gene expression, and they have emerged as pleiotropic, 
highly dysregulated biomarkers in PDAC. We previously demonstrated the feasibility of using microRNAs as prognostic markers in resected PDAC using microRNAs with a demonstrated link to PDAC in the literature. That study was limited due to the single institution design, smaller scope of analyzed microRNAs, and lack of patterns of failure data in validation datasets [13]. In our current study, we evaluated microRNAs most prognostic in a multi-institutional dataset containing survival and patterns of failure data out of all available analyzable microRNAs, thus allowing for a potentially more accurate and generalizable panel. This resulted in the distinct microRNA panel identified in our current study, which identifies a correlation between lower expression of miR$181 \mathrm{~b} / \mathrm{d}$ and miR-575 with increased risk of recurrence. Of these, miR-181b/d has been established as being dysregulated in PDAC versus normal pancreatic tissue and has been linked to gemcitabine resistance $[17,18]$. The exact functions of the miR-181 family in PDAC are unclear, and its role in other malignancies is heterogeneous, being implicated in oncogenesis, cell proliferation, and cell migration by affecting a number of targets [19,20], which could theoretically promote malignant transformation of PDAC and treatment resistance. Additionally, miR-181b has implicated in enhancing chemosensitivity in nonsmall cell lung cancer through inactivation of the TGF $\beta$ R1/Smad signaling pathway [21]. It is possible that decreased expression of $\mathrm{miR}-181 \mathrm{~b}$ promotes hyperactivation of this pathway and confers gemcitabine resistance [22]. Additionally, miR-181d has been associated with tumor suppressive effects in non-small cell lung cancer, as well as in gastric cancer through a variety of targets including PI3K/AKT, a key mediator of disease progression [23,24]. Our findings are consistent with $\mathrm{miR}-181 \mathrm{~b} / \mathrm{d}$ potentially having tumor suppressive roles in PDAC. Although miR-575 has not been previously linked to PDAC, it has been implicated in development of other gastrointestinal cancers, such as gastric cancer via inhibition of PTEN [25], biliary cancer via inhibition of p27Kip1 [26], and hepatocellular carcinoma via inhibition of ST7L [27]. Interestingly, in our study, miR-575 decreased expression was associated with higher risk score and recurrence; it is possible that miR-575 may have disparate tissue-specific functions and that its downregulation is secondary to upregulation of other pathways that mediate PDAC recurrence. Certainly, more studies are needed.

Although radiation was initially supported as adjuvant therapy after prospective evidence of a disease-free and overall survival benefit in a small randomized trial [2], the absence of benefit found in subsequent studies has called into question the benefit of routine adjuvant chemoradiotherapy for resected PDAC [3,4]. Rather, modern practice guidelines recommend consideration of postoperative radiotherapy only in the setting of positive margin (R1) resection [28]. While the role of postoperative chemoradiation is currently being evaluated in the recently completed Radiation Therapy Oncology Group 0848 phase III clinical trial, it is generally accepted that specific subgroups of patients with sufficiently high risk of locoregional recurrence may benefit the most from the addition of radiotherapy [29]. Indeed, many studies suggest potential survival benefit in administering radiation for patients with high-risk clinicopathologic features, such as pathologic lymph node involvement, positive surgical margin status, and elevated CA19-9 [30-34]. Despite increasing awareness of prognostic biomarkers in PDAC [16] and recent genomics-based studies to predict chemotherapeutic response [35,36], there is a dearth of molecular data regarding the utilization of radioresistance biomarkers to predict clinical response to radiotherapy. Given the equivocal role of postoperative radiotherapy for all patients with resected PDAC, which remains associated with high mortality despite improvements in adjuvant chemotherapy regimens [37], a molecular assay-based predictive panel may be useful in selecting subsets of patients for adjuvant radiation. Indeed, similar efforts have led to significant changes in the treatment paradigm for breast cancer, for which the use of molecular assays has become standard of care in the selection of patients for chemotherapy and may be valuable for selection of patients for radiation [38,39]. 
The role of radiotherapy is similarly controversial in locally advanced PDAC, for which the addition of radiation to chemotherapy has not shown an overall survival benefit, with few exceptions [40,41], in the majority of randomized trials [5,42-44]. Additionally, a clear survival benefit to radiotherapy has not been established in patients with resectable or borderline resectable PDAC, as evident in the recent PREOPANC phase III trial (which randomized patients to neoadjuvant chemoradiation versus upfront surgery), despite improvements in lymph node downstaging, reduction in margin positivity rate, and improved disease control from chemoradiotherapy [45]. In the present study, we observed the risk score derived from patients with resected PDAC was also found to be predictive of recurrence and mortality in a cohort of patients who did not receive up front surgery (i.e., borderline-resectable and locally advanced PDAC). Importantly, we found similar locoregional control in patients with a high-risk miRNA score who received radiation and patients with a low-risk score (regardless of radiation), while patients with a high-risk score who did not receive radiotherapy had significantly worse locoregional control, distant recurrence rate, and overall survival (Figure 4). Distant control and overall survival were similarly improved in high-risk patients who received radiotherapy when compared to those who did not, with similar outcomes to those patients in the low-risk cohort. Taken together, selective radiotherapy for patients with high-risk resectable, borderline resectable, or locally advanced PDAC, as determined by the miR risk score, may improve outcomes. Conversely, withholding radiotherapy for low-risk miR risk score patients may spare them unnecessary side effects of radiotherapy.

Strengths of this study include validation on an independent dataset as well as this study representing the largest miRNA profiling effort performed in pancreatic cancer ( $n=183$ patients), with high-quality, clinically annotated data. In addition, the data were acquired from three independent institutions, representing heterogeneous groups of patients that likely better represent the larger patient population diagnosed with PDAC in the U.S and worldwide (i.e., rather than focusing on a single institution). Furthermore, the use of NanoString miRNA profiling platform has advantages that will facilitate broader clinical translation, in that low-input RNA is required, does not require amplification steps by virtue of utilizing direct hybridization, does not require technical replicates, and exhibits high concordance between FFPE and frozen tumor samples. Limitations of the study should also be noted. The study was retrospective in nature and did not include data from prospectively collected patient samples nor from large clinical trials. In addition, the data from borderline resectable and locally advanced PDAC in this study are derived from a single institution with limited patient numbers, and they should be interpreted more cautiously until there is independent validation of the miRNA risk signature in predicting recurrence and mortality in this group of patients.

In conclusion, we developed and validated a promising multi-miRNA risk signature predictive of locoregional recurrence and overall mortality in a multi-institutional cohort of patients with resectable PDAC, and we also validated it an institutional cohort of patients with borderline resectable or locally advanced PDAC. In a secondary analysis of the patients with borderline resectable or locally advanced PDAC (of which about two-thirds received radiation), we found that patients with a high-risk miR score who received radiotherapy likewise had similar locoregional control, distant control, and overall survival as patients with a low-risk score, whereas patients with a high-risk score who did not receive radiotherapy had significantly worse outcomes. This risk score should be further validated prospectively and may be useful in prognosticating PDAC patients and selecting those patients who would benefit from treatment intensification with addition of radiotherapy either adjuvantly or neoadjuvantly.

Supplementary Materials: The following are available online at https:/ / www.mdpi.com/article/10 .3390 / cancers13205168/s1, Table S1: Multivariable analysis for locoregional recurrence in the training cohort of resectable pancreatic cancer patients, Table S2: Multivariable analysis for locoregional recurrence of the validation cohort of resectable pancreatic cancer patients, Table S3: Patient and disease characteristics of patients with borderline resectable or locally advanced pancreatic cancer, 
Table S4: Multivariable analysis for locoregional recurrence of patients with borderline resectable or locally advanced pancreatic cancer.

Author Contributions: Conceptualization, T.M.W.; methodology, N.T.S., T.M.W., A.W., R.R.; software, A.W.; validation, N.T.S., K.W.M., E.J.K., A.W., T.M.W.; formal analysis, N.T.S., T.M.W., A.W.; investigation, N.T.S., A.W., A.R.W., T.J.W.; resources, T.M.W., K.W.M., E.J.K.; data curation, N.T.S., K.W.M., E.J.K., T.M.W., L.Z., D.E., T.J.W., A.R.W., A.W.; writing-original draft preparation, N.T.S., T.M.W., A.W.; writing-review and editing, N.T.S., K.W.M., E.J.K., T.M.W., A.W., L.Z., T.J.W., D.E., R.R., W.C., J.C., M.D., A.T., L.A., A.N.; visualization, N.T.S., A.W., T.M.W.; supervision, T.M.W.; project administration, T.M.W.; funding acquisition, T.M.W., A.R.W. All authors have read and agreed to the published version of the manuscript.

Funding: This work was supported by the following grants: Radiation Oncology Institute Grant (ROI) 2019-913 (T.W./A.W.) and NIH grant R01 CA198128 (TW). Research was also supported through the OSU Gastrointestinal Cancer Research Development fund. Research reported in this article was also supported by The Ohio State University Comprehensive Cancer Center (OSU-CCC) and National Institute of Health (P30 CA016058). Radiation Oncology Institute Grant (ROI) 2019-913 (T.M.W./A.W.).

Institutional Review Board Statement: The study was conducted according to the guidelines of the Declaration of Helsinki, and approved by the Institutional Review Board of the Ohio State University (protocol code 2014C0077 and July 2014).

Informed Consent Statement: Patient consent was waived due to the retrospective nature of this study and due to obtaining consent not being practical from the majority of patients having passed from their disease.

Data Availability Statement: Data can be made available upon request to the corresponding author.

Acknowledgments: We thank P. Fadda in the Genomics Shared Resource at the OSU-CCC for technical support in the nanoString analysis.

Conflicts of Interest: None of the authors have conflicts of interest to disclose.

\section{References}

1. Siegel, R.L.; Miller, K.D.; Jemal, A. Cancer statistics, 2019. CA Cancer J. Clin. 2019, 69, 7-34. [CrossRef]

2. Kalser, M.H.; Ellenberg, S.S. Pancreatic cancer. Adjuvant combined radiation and chemotherapy following curative resection. Arch. Surg. 1985, 120, 899-903. [PubMed]

3. Klinkenbijl, J.H.; Jeekel, J.; Sahmoud, T.; van Pel, R.; Couvreur, M.L.; Veenhof, C.H.; Arnaud, J.P.; Gonzalez, D.G.; de Wit, L.T.; Hennipman, A.; et al. Adjuvant radiotherapy and 5-fluorouracil after curative resection of cancer of the pancreas and periampullary region: Phase III trial of the EORTC gastrointestinal tract cancer cooperative group. Ann. Surg. 1999, 230, 776-782; discussion 782-784, discussion 782-784. [CrossRef]

4. Neoptolemos, J.P.; Stocken, D.D.; Friess, H.; Bassi, C.; Dunn, J.A.; Hickey, H.; Beger, H.; Fernandez-Cruz, L.; Dervenis, C.; La-caine, F.; et al. A randomized trial of chemoradiotherapy and chemotherapy after resection of pancreatic cancer. N. Engl. J. Med. 2004, 350, 1200-1210. [CrossRef]

5. Hammel, P.; Huguet, F.; van Laethem, J.L.; Goldstein, D.; Glimelius, B.; Artru, P.; Borbath, I.; Bouche, O.; Shannon, J.; Andre, T.; et al. Effect of Chemoradiotherapy vs. Chemotherapy on Survival in Patients With Locally Advanced Pancreatic Cancer Controlled After 4 Months of Gemcitabine With or Without Erlotinib: The LAP07 Randomized Clinical Trial. JAMA 2016, 315, 1844-1853. [CrossRef]

6. Katz, M.H.G.; Oncology, F.T.A.F.C.T.O.; Ou, F.-S.; Herman, J.M.; Ahmad, S.A.; Wolpin, B.; Marsh, R.; Behr, S.; Shi, Q.; Chuong, M.; et al. Alliance for clinical trials in oncology (ALLIANCE) trial A021501: Preoperative extended chemotherapy vs. chemotherapy plus hypofractionated radiation therapy for borderline resectable adenocarcinoma of the head of the pancreas. BMC Cancer 2017, 17, 1-8. [CrossRef]

7. Schrag, D. Optimizing Treatment for Locally Advanced Pancreas Cancer: Progress but No Precision. JAMA 2016, 315, 1837-1838. [CrossRef] [PubMed]

8. Sparano, J.A.; Gray, R.J.; Makower, D.F.; Pritchard, K.I.; Albain, K.S.; Hayes, D.F.; Geyer, C.E.; Dees, E.C.; Perez, E.A.; Olson, J.A.; et al. Prospective Validation of a 21-Gene Expression Assay in Breast Cancer. N. Engl. J. Med. 2015, 373, 2005-2014. [CrossRef] [PubMed]

9. Den, R.B.; Yousefi, K.; Trabulsi, E.J.; Abdollah, F.; Choeurng, V.; Feng, F.Y.; Dicker, A.P.; Lallas, C.D.; Gomella, L.G.; Davicio-ni, E.; et al. Genomic classifier identifies men with adverse pathology after radical prostatectomy who benefit from adjuvant radiation therapy. J. Clin. Oncol. 2015, 33, 944-951. [CrossRef] [PubMed]

10. Yonemori, K.; Kurahara, H.; Maemura, K.; Natsugoe, S. MicroRNA in pancreatic cancer. J. Hum. Genet. 2016, 62, 33-40. [CrossRef] 
11. Daoud, A.Z.; Mulholland, E.; Cole, G.; McCarthy, H.O. MicroRNAs in Pancreatic Cancer: Biomarkers, prognostic, and therapeutic modulators. BMC Cancer 2019, 19, 1-13. [CrossRef] [PubMed]

12. Rawat, M.; Kadian, K.; Gupta, Y.; Kumar, A.; Chain, P.S.G.; Kovbasnjuk, O.; Kumar, S.; Parasher, G. MicroRNA in Pancreatic Cancer: From Biology to Thera-peutic Potential. Genes 2019, 10, 752. [CrossRef] [PubMed]

13. Wolfe, A.R.; Wald, P.; Webb, A.; Sebastian, N.; Walston, S.; Robb, R.; Chen, W.; Vedaie, M.; Dillhoff, M.; Frankel, W.L.; et al. A microRNA-based signature predicts local-regional failure and overall survival after pancreatic cancer resection. Oncotarget 2020, 11, 913-923. [CrossRef]

14. Eisenhauer, E.; Therasse, P.; Bogaerts, J.; Schwartz, L.; Sargent, D.; Ford, R.; Dancey, J.; Arbuck, S.; Gwyther, S.; Mooney, M.; et al. New response evaluation criteria in solid tumours: Revised RECIST guideline (version 1.1). Eur. J. Cancer 2009, 45, 228-247. [CrossRef] [PubMed]

15. Pettit, C.; Webb, A.; Walston, S.; Chatterjee, M.; Chen, W.; Frankel, W.; Croce, C.; Williams, T.M. MicroRNA molecular profiling identifies potential signaling pathways conferring resistance to chemoradiation in locally-advanced rectal adenocarcinoma. Oncotarget 2018, 9, 28951-28964. [CrossRef]

16. Hasan, S.; Jacob, R.; Manne, U.; Paluri, R. Advances in pancreatic cancer biomarkers. Oncol. Rev. 2019, 13, 410. [CrossRef]

17. Bloomston, M.; Frankel, W.L.; Petrocca, F.; Volinia, S.; Alder, H.; Hagan, J.P.; Liu, C.G.; Bhatt, D.; Taccioli, C.; Croce, C.M. Mi-croRNA expression patterns to differentiate pancreatic adenocarcinoma from normal pancreas and chronic pancreatitis. JAMA 2007, 297, 1901-1908. [CrossRef]

18. Takiuchi, D.; Eguchi, H.; Nagano, H.; Iwagami, Y.; Tomimaru, Y.; Wada, H.; Kawamoto, K.; Kobayashi, S.; Marubashi, S.; Tanemura, M.; et al. Involvement of microRNA-181b in the gemcitabine resistance of pancreatic cancer cells. Pancreatology 2013, 13, 517-523. [CrossRef]

19. Liu, Y.-S.; Lin, H.-Y.; Lai, S.-W.; Huang, C.-Y.; Huang, B.-R.; Chen, P.-Y.; Wei, K.-C.; Lu, D.-Y. MiR-181b modulates EGFR-dependent VCAM-1 expression and monocyte adhesion in glioblastoma. Oncogene 2017, 36, 5006-5022. [CrossRef]

20. Wang, L.; Wang, Y.X.; Chen, L.P.; Ji, M.L. Upregulation of microRNA-181b inhibits CCL18-induced breast cancer cell metastasis and invasion via the NF-kappaB signaling pathway. Oncol Lett. 2016, 12, 4411-4418. [CrossRef]

21. Wang, X.; Chen, X.; Meng, Q.; Jing, H.; Lu, H.; Yang, Y.; Cai, L.; Zhao, Y. MiR-181b regulates cisplatin chemosensitivity and me-tastasis by targeting TGF $\beta$ R1/Smad signaling pathway in NSCLC. Sci. Rep. 2015, 5, 17618. [CrossRef] [PubMed]

22. Zhuang, J.; Shen, L.; Yang, L.; Huang, X.; Lu, Q.; Cui, Y.; Zheng, X.; Zhao, X.; Zhang, D.; Huang, R.; et al. TGFß1 Promotes Gem-citabine Resistance through Regulating the LncRNA-LET/NF90/miR-145 Signaling Axis in Bladder Cancer. Theranostics 2017, 7, 3053-3067. [CrossRef] [PubMed]

23. Jiang, K.; Xie, L.-F.; Xiao, T.-Z.; Qiu, M.-Y.; Wang, W.-L. MiR-181d inhibits cell proliferation and metastasis through PI3K/AKT pathway in gastric cancer. Eur. Rev. Med. Pharmacol. Sci. 2019, 23, 8861-8869.

24. Conway, J.R.; Herrmann, D.; Evans, T.J.; Morton, J.; Timpson, P. Combating pancreatic cancer with PI3K pathway inhibitors in the era of personalised medicine. Gut 2018, 68, 742-758. [CrossRef]

25. Wang, Y.-N.; Xu, F.; Zhang, P.; Wang, P.; Wei, Y.-N.; Wu, C.; Cheng, S.-J. MicroRNA-575 regulates development of gastric cancer by targeting PTEN. Biomed. Pharmacother. 2019, 113, 108716. [CrossRef]

26. Qin, Y.; Mi, W.; Huang, C.; Li, J.; Zhang, Y.; Fu, Y. Downregulation of miR-575 Inhibits the Tumorigenesis of Gallbladder Cancer via Targeting p27 Kip1. Onco. Targets Ther. 2020, 13, 3667-3676. [CrossRef] [PubMed]

27. Yan, S.; Tang, Z.; Chen, K.; Liu, Y.; Yu, G.; Chen, Q.; Dang, H.; Chen, F.; Ling, J.; Zhu, L.; et al. Long noncoding RNA MIR31HG inhibits hepatocellular carcinoma proliferation and metastasis by sponging microRNA-575 to modulate ST7L expression. J. Exp. Clin. Cancer Res. 2018, 37, 1-16. [CrossRef] [PubMed]

28. National Comprehensive Cancer Network. Pancreatic Cancer (Version 1.2020). Available online: https://www.nccn.org/ professionals / physician_gls/pdf/pancreatic.pdf (accessed on 19 May 2020).

29. Abrams, R.A.; Lowy, A.M.; O’Reilly, E.M.; Wolff, R.A.; Picozzi, V.J.; Pisters, P.W.T. Combined Modality Treatment of Resec-table and Borderline Resectable Pancreas Cancer: Expert Consensus Statement. Ann. Surg. Oncol. 2009, 16, 1751-1756. [CrossRef]

30. Miller, R.C.; Iott, M.J.; Corsini, M.M. Review of Adjuvant Radiochemotherapy for Resected Pancreatic Cancer and Results from Mayo Clinic for the 5th JUCTS Symposium. Int. J. Radiat. Oncol. 2009, 75, 364-368. [CrossRef]

31. Merchant, N.B.; Rymer, J.; Koehler, E.A.; Ayers, G.D.; Castellanos, J.; Kooby, D.A.; Weber, S.H.; Cho, C.S.; Schmidt, C.M.; Na-keeb, A.; et al. Adjuvant chemoradiation therapy for pancreatic adenocarcinoma: Who really benefits? J. Am. Coll. Surg. 2009, 208, 829-838, discussion 838-841. [CrossRef]

32. Hallemeier, C.L.; Botros, M.; Corsini, M.M.; Haddock, M.G.; Gunderson, L.L.; Miller, R.C. Preoperative CA 19-9 Level Is an Important Prognostic Factor in Patients With Pancreatic Adenocarcinoma Treated With Surgical Resection and Adjuvant Concurrent Chemoradiotherapy. Am. J. Clin. Oncol. 2011, 34, 567-572. [CrossRef] [PubMed]

33. Butturini, G.; Stocken, D.D.; Wente, M.N.; Jeekel, H.; Klinkenbijl, J.H.G.; Bakkevold, K.E.; Takada, T.; Amano, H.; Dervenis, C.; Bassi, C.; et al. Influence of Resection Margins and Treatment on Survival in Patients With Pancreatic Cancer: Meta-analysis of Randomized Controlled Trials. Arch. Surg. 2008, 143, 75-83. [CrossRef] [PubMed]

34. Berger, A.C.; Garcia, M., Jr.; Hoffman, J.P.; Regine, W.F.; Abrams, R.A.; Safran, H.; Konski, A.; Benson, A.B., 3rd; MacDonald, J.; Willett, C.G. Postresection CA 19-9 predicts overall survival in patients with pancreatic cancer treated with adjuvant chemoradiation: A prospective validation by RTOG 9704. J. Clin. Oncol. 2008, 26, 5918-5922. [CrossRef] 
35. Aung, K.L.; Fischer, S.E.; Denroche, R.E.; Jang, G.-H.; Dodd, A.; Creighton, S.; Southwood, B.; Liang, S.-B.; Chadwick, D.; Zhang, A.; et al. Genomics-Driven Precision Medicine for Advanced Pancreatic Cancer: Early Results from the COMPASS Trial. Clin. Cancer Res. 2017, 24, 1344-1354. [CrossRef]

36. Connor, A.A.; Denroche, R.E.; Jang, G.H.; Timms, L.; Kalimuthu, S.N.; Selander, I.; McPherson, T.; Wilson, G.W.; Chan-Seng-Yue, M.A.; Borozan, I.; et al. Association of Distinct Mutational Signatures With Correlates of Increased Immune Ac-tivity in Pancreatic Ductal Adenocarcinoma. JAMA Oncol. 2017, 3, 774-783. [CrossRef]

37. Conroy, T.; Hammel, P.; Hebbar, M.; Ben Abdelghani, M.; Wei, A.C.; Raoul, J.-L.; Choné, L.; Francois, E.; Artru, P.; Biagi, J.J.; et al. FOLFIRINOX or Gemcitabine as Adjuvant Therapy for Pancreatic Cancer. N. Engl. J. Med. 2018, 379, 2395-2406. [CrossRef] [PubMed]

38. Sparano, J.A.; Gray, R.J.; Makower, D.F.; Pritchard, K.I.; Albain, K.S.; Hayes, D.F.; Geyer, C.E.; Dees, E.C.; Goetz, M.P.; Olson, J.A.; et al. Adjuvant Chemotherapy Guided by a 21-Gene Expression Assay in Breast Cancer. N. Engl. J. Med. 2018, 379, 111-121. [CrossRef]

39. Parulekar, W.R.; Berrang, T.; Kong, I.; Rakovitch, E.; Theberge, V.; Gelmon, K.A.; Chia, S.K.L.; Bellon, J.R.; Jagsi, R.; Ho, A.Y.; et al. Cctg MA.39 tailor RT: A randomized trial of regional radiotherapy in biomarker low-risk node-positive breast cancer (NCT03488693). J. Clin. Oncol. 2019, 37, TPS602. [CrossRef]

40. Group, G.T.S. Treatment of locally unresectable carcinoma of the pancreas: Comparison of combined-modality therapy (chem-otherapy plus radiotherapy) to chemotherapy alone. Gastrointestinal Tumor Study Group. J. Natl. Cancer Inst. 1988, 80, 751-755.

41. Sr, P.J.L.; Feng, Y.; Cardenes, H.; Wagner, L.; Brell, J.M.; Cella, D.; Flynn, P.; Ramanathan, R.K.; Crane, C.H.; Alberts, S.R.; et al. Gemcitabine Alone Versus Gemcitabine Plus Radiotherapy in Patients With Locally Advanced Pancreatic Cancer: An Eastern Cooperative Oncology Group Trial. J. Clin. Oncol. 2011, 29, 4105-4112.

42. Hazel, J.J.; Thirlwell, M.P.; Huggins, M.; Maksymiuk, A.; MacFarlane, J.K. Multi-drug chemotherapy with and without radiation for carcinoma of the stomach and pancreas: A prospective randomized trial. J. Can. Assoc. Radiol. 1981, 32, 164-165. [PubMed]

43. Klaassen, D.J.; MacIntyre, J.M.; Catton, G.E.; Engstrom, P.F.; Moertel, C.G. Treatment of locally unresectable cancer of the stomach and pancreas: A randomized comparison of 5-fluorouracil alone with radiation plus concurrent and maintenance 5-fluorouracil-an Eastern Cooperative Oncology Group study. J. Clin. Oncol. 1985, 3, 373-378. [CrossRef]

44. Chauffert, B.; Mornex, F.; Bonnetain, F.; Rougier, P.; Mariette, C.; Bouché, O.; Bosset, J.F.; Aparicio, T.; Mineur, L.; Azzedine, A.; et al. Phase III trial comparing intensive induction chemoradiotherapy (60 Gy, infusional 5-FU and intermittent cisplatin) followed by maintenance gemcitabine with gemcitabine alone for locally advanced unresectable pancreatic cancer. Definitive results of the 2000-01 FFCD/SFRO study. Ann. Oncol. 2008, 19, 1592-1599. [PubMed]

45. Versteijne, E.; Suker, M.; Groothuis, K.; Akkermans-Vogelaar, J.M.; Besselink, M.G.; Bonsing, B.A.; Buijsen, J.; Busch, O.R.; Creemers, G.M.; van Dam, R.M.; et al. Preoperative Chemoradiotherapy Versus Immediate Surgery for Resectable and Border-line Resectable Pancreatic Cancer: Results of the Dutch Randomized Phase III PREOPANC Trial. J. Clin. Oncol. 2020, 38, 1763-1773. [CrossRef] [PubMed] 